\title{
Change in acoustic radiation force impulse is a better indicator of liver disease progression or regression
}

\author{
Hoie K Leong ${ }^{1,2 *}$, Robert Gibson ${ }^{2,3}$, Siddharth Sood ${ }^{1,2}$, David Nadebaum ${ }^{1}$, Alexandra Gorelik ${ }^{2,4}$ and Amanda J Nicoll1 $^{2,5,6}$ \\ ${ }^{1}$ Department of Gastroenterology and Hepatology, Royal Melbourne Hospital, Melbourne, Australia \\ ${ }^{2}$ University of Melbourne, Melbourne, Australia \\ ${ }^{3}$ Department of Radiology, Royal Melbourne Hospital, Melbourne, Australia \\ ${ }^{4}$ Melbourne Epicentre, Royal Melbourne Hospital, Melbourne, Australia \\ ${ }^{5}$ Department of Gastroenterology, Eastern Health, Melbourne, Australia \\ ${ }^{6}$ Monash University, Melbourne, Australia
}

\begin{abstract}
Objective: To investigate whether the change in Acoustic Radiation Force Impulse (ARFI) readings over time has value in assessing the clinical trajectory of patients with chronic liver disease.

Design: All chronic liver disease patients with at least two ARFI liver stiffness measurements between August 2012-March 2016 were included in our study. Demographics, etiology of liver disease, BMI, blood test results, presence of cirrhosis and liver complications were obtained at baseline and follow up. The percentage change in ARFI scores per year was calculated for each patient. Initiation of any treatment likely to improve liver disease, and clinical features of decompensation of the liver disease, between ARFI measurements were documented. Patients with less than 6 months between baseline and follow up ARFI, or an IQR: Median velocity ratio $>0.30$ (indicative of unreliable readings) were excluded from the study.

Results: one hundred and twelve (112) patients met the inclusion criteria and were analyzed in the study. Patients who developed new varices (median \% ARFI change $17.3, p=0.01$ ) or experienced a new drug-induced liver injury (median \% ARFI change 21.6, $p=0.01$ ) had significant increases in percent change in ARFI/ yr, while new HCV treatment (median \% ARFI change $-19.1, p=0.04$ ) was associated with significant decrease. There was no statistically significant difference with new HBV treatment (median \% ARFI change $-17.2, p=0.14$ ) and alcohol cessation (median \% ARFI change $-0.67, p=0.71$ ).
\end{abstract}

Conclusion: Percentage change in ARFI may be a better clinical indicator of disease prognosis or regression than absolute values, as it may account for individual confounding variables.

Abbreviations: ARFI: Acoustic Radiation Force Impulse; FIB4: Fibrosis 4 Index; APRI: Aspartate Aminotransferase-toPlatelet Ratio Index; AST: Aspartate Aminotranferase; ALT: Alanine Aminotransferase; AST: to ALT ratio; LSM: Liver Stiffness Measurements; HC: Hepatocellular Carcinoma; AASLD: American Association for the Study of Liver Diseases; BMI: Body Mass Index; DILI: Drug-Induced Liver Injury; IQR: Interquartile Range

\section{Introduction}

Chronic liver disease is characterized by the progressive development of fibrosis and determining the degree of fibrosis is important in guiding disease management and prognosis [1]. The complications that occur in chronic liver disease are associated with significant morbidity and mortality, and correlate with the degree of liver fibrosis. Traditionally, liver biopsy has been the gold standard in assessing fibrosis and has shown good accuracy in determining prognosis [2]. However, liver biopsy has limitations, as it is an invasive procedure with a small risk of serious complications, and the accuracy can suffer from intra-observer variability, inter-observer variability and sampling error [3]. For this reason, very few patients have serial liver histology information to determine the disease progression or response to therapy.
A well validated non-invasive method of estimating fibrosis is Acoustic Radiation Force Impulse (ARFI) Imaging. This term includes all techniques that utilizes the force of an ultrasound beam at a focal point to generate shear waves. ARFI measures liver stiffness by delivering narrow high energy ultrasound pulses that result in slight tissue displacements, which in turn generate low amplitude shear waves that travel in a lateral direction away from the line of the ultrasound beam. Ultrasound pulses are used to track shear wave propagation; with shear wave velocity shown to positively correlate with tissue stiffness and liver fibrosis [4-6].

Clinically, interpretation of ARFI liver stiffness measurements (LSM) are commonly based on absolute values derived from pooled meta analyses. One example groups ARFI LSM values such that $<1.35$ $\mathrm{m} / \mathrm{s}=\mathrm{F} 0-\mathrm{F} 1,1.35-1.54 \mathrm{~m} / \mathrm{s}=\mathrm{F} 2,1.55-1.80 \mathrm{~m} / \mathrm{s}=\mathrm{F} 3$ and $>1.80 \mathrm{~m} / \mathrm{s}$

*Correspondence to: Hoie Kidd Leong, Department of Gastroenterology and Hepatology, Royal Melbourne Hospital, Melbourne, Australia, Tel: 61433796 086; E-mail: kiddleong5@gmail.com

Key words: acoustic radiation force impulse (ARFI), shear wave elastography, chronic liver disease, fibrosis, liver

Received: July 04, 2018; Accepted: July 23, 2018; Published: July 27, 2018 
= F4(4), where F0 is normal liver, F1 is mild fibrosis, F2 is moderate fibrosis, F3 is severe fibrosis (pre-cirrhotic), and F4 is cirrhosis as determined on histology by Scheuer or Metavir scores. Previous studies have investigated whether absolute ARFI values can predict complications or improvement in patients with chronic liver disease, with conflicting results [7-9].

To our knowledge, the change in serial ARFI measurements has not been studied as a method of assessing patient outcomes in patients with chronic liver disease. The aim of this study is to investigate whether the percentage change in ARFI velocity over time showed additional prognostic benefit above that of single absolute ARFI measurements.

\section{Methods}

All patients that completed at least two ARFI LSM at our institution between August 2012 and March 2016 were included in the study. Baseline patient information was obtained from medical records at the time of initial ARFI assessment. This information included demographic data, etiology of liver disease and presence of liver disease complications. Baseline pathology results were also recorded and used for the calculation of Fibrosis 4 Index (FIB4), aspartate aminotransferase-to-platelet ratio index (APRI) and aspartate aminotransferase (AST) to alanine aminotransferase (ALT) ratio. The days between the baseline ARFI LSM and pathology results was recorded. Cirrhosis was diagnosed by liver histology and/or based on results of clinical, laboratory and imaging studies as per the accepted guidelines [10]. If cirrhosis was present, Child Pugh Score and MELD scores were calculated. The presence of varices was diagnosed via upper gastrointestinal endoscopy. The presence of ascites required either ultrasound detected or clinically detected ascites. Encephalopathy was present if it was clinically documented by a consultant hepatologist that the patient had encephalopathy. Hepatocellular carcinoma (HCC) was determined by typical features seen on quad phase CT or MRI according to the American Association for the Study of Liver Diseases guidelines [11] or biopsy proven HCC.

At the follow up ARFI LSM, the change in ARFI LSM, change in ARFI LSM per year, percentage change and percentage change per year were calculated. A follow up body mass index (BMI) was also recorded if available. Medical records were then searched again for biochemistry data closest to the date of the follow up ARFI LSM and follow up FIB4, APRI and AST:ALT ratios were calculated. Albumin and bilirubin closest to the follow up ARFI were also documented. For albumin, bilirubin and each serological formula, a change between baseline and follow up measurement and change between baseline and follow up measurement per year were calculated. Medical records were also searched for liver decompensation event data for the duration between the baseline and follow up ARFI LSM. The occurrence of clinical 'events' between baseline and follow-up ARFI examinations was determined from medical records. Events recorded included the development of esophageal varices, upper gastrointestinal bleeding, ascites, encephalopathy, HCC or episodes of drug-induced liver injury. In patients with alcoholic liver disease, data was collected on whether the patient became abstinent from alcohol between the two ARFI measurements. In patients that had hepatitis B or hepatitis C, data was collected on whether treatment for these conditions were started during the follow up period.

\section{ARFI procedure}

ARFI measurements were acquired with the Siemens Acuson S2000 ultrasound system by at least two different sonographers experienced with this technique. Patients were fasted for at least 6 hours and measurements were taken with the patient in the supine position. A region of interest was set in the right hepatic lobe through an intercostal approach two to four centimeters below the liver capsule. The region of interest was placed in an area of parenchyma that was free of blood vessels. Measurements were taken by each sonographer until 10 successful LSM were obtained. The average of the median LSM from both sonographers were used as the ARFI measurement for this study. The inter-observer variability has been described previously [12]. Mean, standard deviations, interquartile range (IQR) and IQR/Median velocity ratio of the ARFI measurements were also provided by the ARFI ultrasound system. The IQR is calculated using all the successful LSM measurements obtained by each sonographer. This value divided by the average of the median LSM from both sonographers gives the IQR/Median velocity ratio. It has been shown that ARFI measurements with an IQR/Median velocity ratio of $>0.30$ has reduced accuracy in staging fibrosis [13].

\section{Calculation of serological formulas}

FIB4, APRI and AST:ALT ratio was calculated. The scores have all been previously correlated to the Metavir system of histological changes of fibrosis and stages fibrosis into F0 to F4. FIB4 was calculated as previously described such that a FIB4 score of $<1.45$ is consistent with a fibrosis score of $\leq \mathrm{F} 2$, while a score of $>3.25$ is consistent with a fibrosis score of $\geq$ F3. A score between 1.45 and 3.25 is considered the indeterminate range [14]. The APRI score was calculated and a threshold APRI score of 1.5 has a $93 \%$ specificity for significant fibrosis, while an APRI score of 2.0 has a specificity of $93 \%$ for severe fibrosis and $91 \%$ for cirrhosis [15]. An AST to ALT ratio of between 0.6-1.0 is suggestive of a fibrosis stage of F2-F3, while a score of $>1.0$ is highly suggestive of cirrhosis.

\section{Exclusion criteria}

Patients with a time between ARFI measurements of less than 6 months were excluded from the study, as six months was felt to be of appropriate minimum duration to allow clinically meaningful disease progression/regression. In addition, because it has been shown that ARFI measurements with an IQR/Median velocity of $>0.30$ has a reduced accuracy [13], patients with either a baseline or follow up ARFI measurement with an IQR: median velocity of $>0.30$ were excluded. Patients with an ALT or AST greater than five times the upper limit of normal were excluded as this can affect the accuracy of ARFI Liver stiffness measurements.

\section{Statistical analysis}

Statistical analyses were performed using Stata 12 [StataCorp. Stat Statistical software. Release 12. College Station, TX]. Correlations between percent change in ARFI per year and change in albumin, bilirubin, FIB4, APRI and AST:ALT ratio per year was calculated using Spearman's rank correlation coefficient. To calculate if there were statistical differences between patients that experienced "events" opposed to those who didn't when comparing their percent change in ARFI per year scores, a two-sample Wilcoxon rank-sum (MannWhitney) test was used. This test was also used to determine if there was any statistical significance in the change in serological formulas per year.

\section{Ethics and consent}

This study was approved by The Melbourne Health, Office for Research, Human Ethics committee as a quality assurance project. 
Verbal consent was obtained by the doctor booking the ultrasound. According to our ethics committee, no written consent was required as this was deemed a low risk project.

\section{Results}

Two hundred and thirteen patients had at least two ARFI LSM. Eight patients were excluded because they had ARFI readings less than six months apart. A further 93 patients were excluded because they had either a first ARFI or second ARFI that had poor reliability, as determined by an IQR/Median velocity ratio $>0.30$ [13]. The patient cohort is shown in Figure 1. One hundred and twelve patients were included in the study and their baseline and follow up characteristics are shown in Table 1 . Of these 112 patients, 50 (45\%) were male and the median age was 49.5 years (range 18-74). 19 patients had cirrhosis at baseline. The median days between the baseline and follow up ARFI was 554 days with an IQR of 351.25 days. The BMI of all patients at baseline showed that the majority had normal BMI (53\%, with $37 \%$ in the overweight range). Only a small number were in the obese category (10\%).
Table 2 shows the median percentage change per year in ARFI for patients who had events during follow up, compared to those who did not. There were statistically significant differences in those that developed varices, started a new hepatitis $C$ treatment or experienced a new drug-induced liver injury. There were no statistically significant differences between those who started a new hepatitis B treatment or ceased alcohol during the follow up period. No cases of new ascites, hepatic encephalopathy or HCC were seen.

Table 3 shows the characteristics of patients based on whether their ARFI LSM per year suggested progression, stable or improved liver disease. Seventeen patients had a greater than ten per cent increase in ARFI LSM/year, suggesting progression of their liver disease. Of these 17 , three developed varices (18\%), and three had a new drug-induced liver injury (18\%) between the two measurements. None started antiviral therapy for hepatitis B or hepatitis $\mathrm{C}$, and none with alcoholic liver disease became abstinent during this period. Twenty-five patients had a greater than ten per cent decrease in AFRI LSM/year, suggesting an improvement in liver disease. Of this 25 , none developed varices or

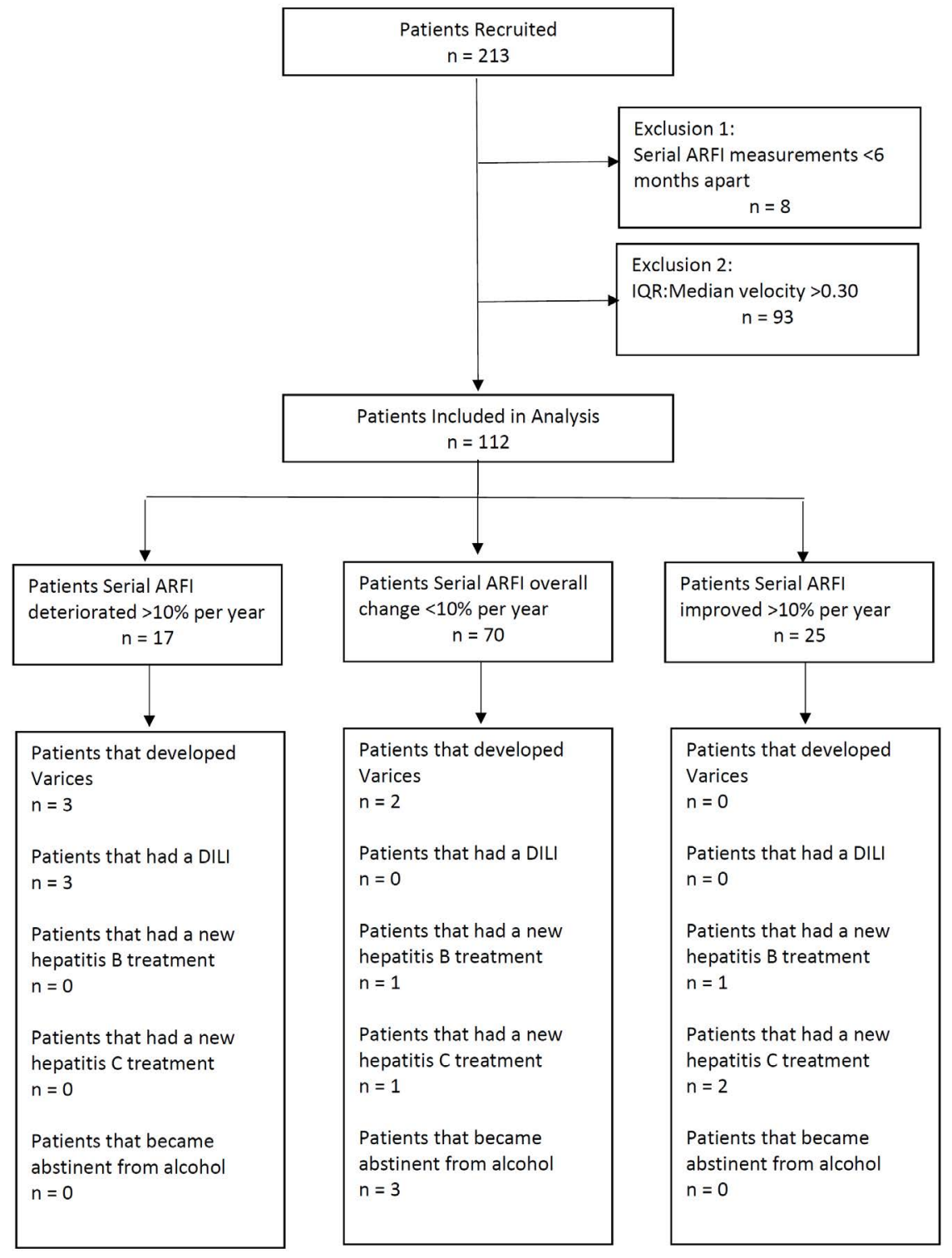

Figure 1. Flow diagram of cohort 
Table 1. Baseline Characteristics of the cohort including evidence of liver decompensation

\begin{tabular}{|c|c|c|}
\hline Characteristic & Baseline & Follow Up \\
\hline Number & 112 & 112 \\
\hline Age & $\begin{array}{l}49.50 \pm 23.25 \\
(47.20,14.55)\end{array}$ & $\begin{array}{l}51.20 \pm 22.45 \\
(48.45,14.68)\end{array}$ \\
\hline \multicolumn{3}{|l|}{ median \pm IQR (mean, standard deviation) } \\
\hline Male: Female & $50(45 \%): 62(55 \%)$ & $50(45 \%): 62(55 \%)$ \\
\hline BMI $<25 \mathrm{~kg} / \mathrm{m}^{2}: 25-30 \mathrm{~kg} / \mathrm{m}^{2}:>30 \mathrm{~kg} / \mathrm{m}^{2}$ & $59: 41: 11$ & $52: 31: 03$ \\
\hline \multicolumn{3}{|l|}{ Aetiology } \\
\hline HBV & $36(32 \%)$ & $36(32 \%)$ \\
\hline $\mathrm{HCV}$ & $23(21 \%)$ & $23(21 \%)$ \\
\hline NAFLD & $20(18 \%)$ & $20(18 \%)$ \\
\hline Alcohol & $10(9 \%)$ & $10(9 \%)$ \\
\hline Other & $40(36 \%)$ & $40(36 \%)$ \\
\hline Clinical Evidence of Cirrhosis & $19(17 \%)$ & \\
\hline Albumin $-\mathrm{g} / \mathrm{L}$ & $39(6.00)$ & $39(5.00)$ \\
\hline \multicolumn{3}{|l|}{ median (IQR) } \\
\hline Bilirubin - micromol/L & $11.5(7.75)$ & $11.5(5.00)$ \\
\hline \multicolumn{3}{|l|}{ median (IQR) } \\
\hline FIB4 & $1.36(1.24)$ & $1.34(1.60)$ \\
\hline \multicolumn{3}{|l|}{ median (IQR) } \\
\hline APRI & $0.54(0.52)$ & $0.50(0.50)$ \\
\hline \multicolumn{3}{|l|}{ median (IQR) } \\
\hline AAR & $1.00(0.53)$ & $1.04(0.59)$ \\
\hline \multicolumn{3}{|l|}{ median (IQR) } \\
\hline Varices & $9(8 \%)$ & $14(13 \%)$ \\
\hline Ascites & $3(3 \%)$ & $3(3 \%)$ \\
\hline Encephalopathy & $2(2 \%)$ & $2(2 \%)$ \\
\hline $\mathrm{HCC}$ & $1(1 \%)$ & $1(1 \%)$ \\
\hline \multicolumn{3}{|l|}{ ARFI LSM in m/s (median) } \\
\hline$<1.35 \mathrm{~m} / \mathrm{s}-$ absent or mild fibrosis (F0 or F1) & 58 & 64 \\
\hline $1.35-1.54 \mathrm{~m} / \mathrm{s}-$ significant fibrosis $(\mathrm{F} 2)$ & 25 & 21 \\
\hline $1.55-1.80 \mathrm{~m} / \mathrm{s}-$ severe fibrosis $(\mathrm{F} 3)$ & 10 & 11 \\
\hline$>1.80 \mathrm{~m} / \mathrm{s}-$ cirrhosis $(\mathrm{F} 4)$ & 19 & 16 \\
\hline
\end{tabular}

IQR: Interquartile range; HBV: Hepatitis B; HCV: Hepatitis C; NAFLD: Non-alcoholic fatty liver disease.

Table 2. Percentage change in ARFI for patients that had liver decompensation events or started therapy likely to improve the underlying liver disease. This shows that the median ARFI had significantly increased in those who developed varices and had significan decreased in those who had treatment for hepatitis $\mathrm{C}$ or had resolving drug induced liver disease. Treatment of HBV and alcohol abstinence did not show any significant difference

\begin{tabular}{|l|l|l|}
\hline New event between ARFI scans & Median \% change ARFI/yr (range) & $\boldsymbol{p}$ value \\
\hline Varices $(\mathrm{n}=5)$ & $17.3(6.1$ to 24.4$)$ & 0.01 \\
\hline HBV Treatment $(\mathrm{n}=2)$ & $-17.2(-19.1$ to -4.9$)$ & 0.14 \\
\hline HCV Treatment $(\mathrm{n}=3)$ & $-19.1(-30.7$ to -3.8$)$ & 0.04 \\
\hline Alcohol Abstinence $(\mathrm{n}=3)$ & $-0.67(-1.0$ to 6.1$)$ & 0.71 \\
\hline DILI $(\mathrm{n}=3)$ & $21.6(19.0$ to 44.7$)$ & 0.01 \\
\hline
\end{tabular}

had a drug-induced liver injury, but three did start antiviral therapy (12\%) (1 for hepatitis B, 2 for hepatitis C). No patient in this group became abstinent from alcohol during this period. Seventy patients had less than ten per cent change in ARFI LSM per year. Only two of these 70 (3\%) developed varices, and two started antiviral therapy (3\%) (1 for hepatitis B, 1 for hepatitis C). Three of these patients became abstinent from alcohol between the two ARFI measurements. No patients developed ascites, encephalopathy, HCC or had a new treatment for autoimmune hepatitis between baseline and follow up ARFI LSM.

Most pathology blood tests on which the serological values were calculated were done on the same day as the ultrasound and ARFI examination. The median time between the date of the full blood examination (FBE), and the baseline and follow up ARFI measurements was $5.00 \pm 47.50$ days and $0.00 \pm 20.00$ days respectively. The median number of days ( \pm IQR) between the date of the liver function tests (LFT) and the baseline and second ARFI measurements was $0.00 \pm$ 28.00 and $0.00 \pm 22.50$ respectively. Albumin and bilirubin values at baseline and follow up are shown in table 1.

To determine if the change in ARFI LSM per year was mirrored by the pathology results, BMI or serological markers of liver fibrosis, the correlation coefficient was calculated using Spearman's test. This is shown in table 4. This shows that the change in ARFI LSM over time correlated with the change in FIB4 and APRI; however, no significant correlation was seen with the change in serum albumin, bilirubin, BMI, or AAR.

\section{Discussion and conclusion}

This study is the first to our knowledge to demonstrate that the percentage change in ARFI LSM over time does correlate with clinical progression or improvement in liver disease. Single time point ARFI measurements are influenced by several factors including BMI, body habitus, liver steatosis and inflammation [13,16-18], as well as the amount of fibrosis. In addition, our previous research has shown that single time point ARFI measurements did not correlate with complications in patients with cirrhosis [19]. However, in an individual patient, factors that affect ARFI accuracy and its relationship to underlying fibrosis remain relatively stable. Therefore, serial changes in an individual may be more reflective of changes in fibrosis and progression or regression of liver damage. In this study, five different liver decompensation "events" were seen during the follow up period. Serial ARFI LSM increased overtime in patients who developed new varices, a new drug-induced liver injury and decreased over time in those who started anti-viral therapy during the follow up period. There was no correlation found in patients that ceased alcohol during the follow up period.

Patients that developed new varices within the follow up period had increasing serial ARFI LSM with a mean change of $15.4 \%$ per year $(p=0.01)$. This is consistent with the hypothesis that as chronic liver disease progresses, there is increasing fibrosis leading to increasing liver stiffness that is detectable by ARFI. Patients that developed a drug-induced liver injury during the follow up period showed the same deterioration in ARFI LSM with a mean percentage change per year of $28.4(p=0.01)$. Drug-induced liver injury tends to be more of an acute event and this finding may be confounded by the fact that the follow up ARFI LSM would have been done close to the time of their drug-induced liver injury, where acute inflammatory processes may be responsible for the increase in ARFI LSM.

Treatment of viral hepatitis has been shown to improve the histology with a reduction in inflammation and fibrosis occurring [20], and a reduction in liver disease complications. It would therefore be expected that treatment of viral hepatitis would result in an improvement in the ARFI LSM over the median time of almost 2 years ( 554 days). This was demonstrated in our study, with patients who received a new hepatitis $\mathrm{B}$ or hepatitis $\mathrm{C}$ treatment during follow up showing improvements in their ARFI LSM, with mean percentage change per year being -17.2 ( $p$ $=0.14)$ and $-19.1(p=0.04)$ respectively. Only patients that had a new HCV treatment had a statistically significant change in ARFI LSM.

Our study found no correlation with abstinence from alcohol and serial ARFI LSM. Only ten patients with alcohol related liver disease were included, of which three became abstinent from alcohol. Two had very high measurements at baseline and follow up, and this may 
Table 3. Comparison of Patients with Progression of Liver Disease, stable Liver Disease and Improved Liver disease as determined by a greater than $10 \%$ change in ARFI LSM

\begin{tabular}{|c|c|c|c|c|}
\hline & $\begin{array}{l}\text { Progression of Liver Disease } \\
\text { (ARFI LSM > } 10 \% \text { higher) }\end{array}$ & Stable Liver Disease & Improved Liver disease & $p$ \\
\hline & & (ARFI < 10\% change) & (ARFI LSM $>10 \%$ lower) & \\
\hline Number & 17 & 70 & 25 & \\
\hline Age & 55 & 49 & 48 & 0.627 \\
\hline - Median (IQR) & (38 to 57 ) & (33 to 57 ) & (37 to 62 ) & \\
\hline Male: Female & 8:09 & $30: 40: 00$ & $12: 13$ & 0.891 \\
\hline \multicolumn{5}{|l|}{ BMI baseline } \\
\hline$<25 \mathrm{~kg} / \mathrm{m}^{2}: 25-30 \mathrm{~kg} / \mathrm{m}^{2}:>30 \mathrm{~kg} / \mathrm{m}^{2}$ & 10:06:01 & $39: 23: 07$ & 10:12:03 & 0.637 \\
\hline \multicolumn{5}{|l|}{ BMI follow up } \\
\hline$<25 \mathrm{~kg} / \mathrm{m}^{2}: 25-30 \mathrm{~kg} / \mathrm{m}^{2}:>30 \mathrm{~kg} / \mathrm{m}^{2}$ & 9:08:00 & $36: 16: 03$ & 7:07:00 & 0.431 \\
\hline Reduction in BMI & $8(47 \%)$ & $40(57 \%)$ & $14(56 \%)$ & 0.753 \\
\hline Type of liver disease & $6: 3: 2: 2: 7$ & $23: 16: 11: 7: 23$ & $7: 4: 7: 1: 10$ & 0.89 \\
\hline \multicolumn{5}{|l|}{ HBV:HCV:NAFLD:Alc:other } \\
\hline HBV or HCV Treatment started & $0(0 \%)$ & $2(3 \%)$ & $3(12 \%)$ & \\
\hline Change in FIB4 & 0.12 & 0.02 & -0.13 & 0.356 \\
\hline - Median (IQR) & $(-0.21$ to 0.61$)$ & $(-0.25$ to 0.33$)$ & \multicolumn{2}{|c|}{$(-0.64$ to 0.18$)$} \\
\hline Change in APRI & -0.01 & -0.02 & -0.08 & 0.052 \\
\hline - Median (IQR) & $(-0.13$ to 0.38$)$ & $(-0.12$ to 0.08$)$ & \multicolumn{2}{|c|}{$(-0.67$ to -0.02$)$} \\
\hline Change in AST:ALT & 0.02 & -0.03 & 0.03 & 0.819 \\
\hline - Median (IQR) & $(-0.18$ to 0.13$)$ & $(-0.31$ to 0.23$)$ & \multicolumn{2}{|c|}{$(-0.18$ to 0.17$)$} \\
\hline Evidence of liver disease progression & $6(35 \%)$ & $7(10 \%)$ & $3(12 \%)$ & 0.04 \\
\hline Developed varices & $3(18 \%)$ & $2(3 \%)$ & $0(0 \%)$ & 0.034 \\
\hline New DILI & $3(18 \%)$ & $0(0 \%)$ & $0(0 \%)$ & 0.003 \\
\hline Started HBV anti-viral & $0(0 \%)$ & $1(1 \%)$ & $1(4 \%)$ & 0.611 \\
\hline Started HCV treatment* & $0(0 \%)$ & $1(1 \%)$ & $2(8 \%)$ & 0.184 \\
\hline Alcohol abstinence & $0(0 \%)$ & $3(4 \%)$ & $0(0 \%)$ & 0.735 \\
\hline
\end{tabular}

*Hepatitis $\mathrm{C}$ was treated with pegylated interferon plus ribavirin as direct acting anti-viral were not available in Australia until after recruitment finished.

Table 4. Correlations between percent change in ARFI and changes in biochemical markers. This shows the Spearman's correlation coefficients for the relationship between the percentage change in ARFI LSM per year and each individual change per year for the serological markers or formulas

\begin{tabular}{|c|c|c|}
\hline $\begin{array}{c}\text { Change in Marker or } \\
\text { Serological Formula per year }\end{array}$ & Spearman's coefficient & $\boldsymbol{p}$ value \\
\hline Albumin & -0.03 & 0.85 \\
\hline Bilirubin & -0.21 & 0.14 \\
\hline FIB4 & 0.21 & 0.06 \\
\hline APRI & 0.23 & 0.04 \\
\hline AST: ALT & 0.13 & 0.25 \\
\hline
\end{tabular}

reflect established cirrhosis that was unlikely to change, and the third had normal liver stiffness at baseline. There is limited evidence that abstinence causes regression of fibrosis in those with well-established cirrhosis [21].

The serological markers of fibrosis, FIB4, APRI and AST to ALT ratio, have been useful in excluding cirrhosis in at risk patients [22-24]. In this study, we examined if they changed in concordance with ARFI LSM. The change in FIB4 and APRI correlated with the change in ARFI LSM. The AST to ALT ratio did not correlate and this may reflect that this is an insensitive tool for liver disease progression or regression [25]. It is likely in the future that a combination of elastography and serological tools will be used to guide management, but how to combine the information from both sets of tests is still in evolution [26].

The main limitation of this study was that there were a low number of liver decompensation events during follow up (16 events in total), which makes it difficult to make strong conclusions about the findings in this study. There were also many patients excluded due to poor reproducibility of the ARFI readings as represented by an IQR/Median velocity $>0.30$. Follow up was a median of 18 months and longer follow up should better reflect if the change in ARFI LSM was a predictor of outcome.
Liver stiffness measurements using ARFI are a useful tool in the management of liver disease and are particularly useful to research where the cohort effect can be seen. However, in an individual patient the single point measurement may be inaccurate, but the greater value may be in the change in each individual patient over time. The future of all liver shear wave technologies will need to account for confounders such as body habitus and using the change in serial measurements may be much more valuable.

\section{Funding}

Australian Post Graduate Award Scholarship, University of Melbourne.

\section{References}

1. Lefton HB, Rosa A, Cohen M (2009) Diagnosis and epidemiology of cirrhosis. Med Clin North Am 93: 787-799, vii. [Crossref]

2. Dienstag JL (2002) The role of liver biopsy in chronic hepatitis C. Hepatology 36: S152-S160. [Crossref]

3. Bravo AA, Sheth SG, Chopra S (2001) Liver biopsy. NEngl J Med 344: 495-500. [Crossref]

4. Friedrich-Rust M, Nierhoff J, Lupsor M, Sporea I, Fierbinteanu-Braticevici C, et al. (2012) Performance of Acoustic Radiation Force Impulse imaging for the staging of liver fibrosis: a pooled meta-analysis. J Viral Hepat 19: e212-e219. [Crossref]

5. Bota S, Herkner H, Sporea I, Salzl P, Sirli R, et al. (2013) Meta-analysis: ARFI elastography versus transient elastography for the evaluation of liver fibrosis. Liver Int 33: 1138-1147. [Crossref]

6. Yap WW, Kirke R, Yoshida EM, Owen D, Harris AC (2013) Non-invasive assessment of liver fibrosis using ARFI with pathological correlation, a prospective study. Ann Hepatol 12: 608-615.

7. Rizzo L, Calvaruso V, Cacopardo B, Alessi N, Attanasio M, et al. (2011) Comparison of transient elastography and acoustic radiation force impulse for non-invasive staging of liver fibrosis in patients with chronic hepatitis C. Am J Gastroenterol 106: 2112-2120. [Crossref] 
8. Vermehren J, Polta A, Zimmermann O, Herrmann E, Poynard T, et al. (2012) Comparison of acoustic radiation force impulse imaging with transient elastography for the detection of complications in patients with cirrhosis. Liver Int 32: 852-858. [Crossref]

9. Mori K, Arai H, Abe T, Takayama H, Toyoda M, et al. (2013) Spleen stiffness correlates with the presence of ascites but not esophageal varices in chronic hepatitis $\mathrm{C}$ patients. Biomed Res Int: 857862 .

10. Bellentani S, Tiribelli C, Saccoccio G, Sodde M, Fratti N, et al. (1994) Prevalence of chronic liver disease in the general population of northern Italy: the Dionysos Study. Hepatology 20: 1442-1449. [Crossref]

11. Bruix J, Sherman M; American Association for the Study of Liver Diseases (2011) Management of hepatocellular carcinoma: an update. Hepatology 53: 1020-1022. [Crossref]

12. Nadebaum DP, Nicoll AJ, Sood S, Gorelik A, Gibson RN (2018) Variability of Liver Shear Wave Measurements Using a New Ultrasound Elastographic Technique. $J$ Ultrasound Med 37: 647-656. [Crossref]

13. Bota S, Sporea I, Sirli R, Popescu A, Jurchis A (2013) Factors which influence the accuracy of acoustic radiation force impulse (ARFI) elastography for the diagnosis of liver fibrosis in patients with chronic hepatitis C. Ultrasound Med Biol 39: 407-412. [Crossref]

14. Vallet-Pichard A, Mallet V, Nalpas B, Verkarre V, Nalpas A, et al. (2007) FIB-4: an inexpensive and accurate marker of fibrosis in $\mathrm{HCV}$ infection. comparison with liver biopsy and fibrotest. Hepatology 46: 32-36. [Crossref]

15. Lin ZH, Xin YN, Dong QJ, Wang Q, Jiang XJ, et al. (2011) Performance of the aspartate aminotransferase-to-platelet ratio index for the staging of hepatitis C-related fibrosis: an updated meta-analysis. Hepatology 53: 726-736. [Crossref]

16. Arena U, Vizzutti F, Abraldes JG, Corti G, Stasi C, et al. (2008) Reliability of transient elastography for the diagnosis of advanced fibrosis in chronic hepatitis C. Gut 57: 12881293. [Crossref]
17. Petta S, Di Marco V, Camma C, Butera G, Cabibi D, Craxi A (2011) Reliability of liver stiffness measurement in non-alcoholic fatty liver disease: the effects of body mass index. Aliment Pharmacol Ther 33: 1350-1360. [Crossref]

18. Gaia S, Carenzi S, Barilli AL, Bugianesi E, Smedile A, et al. (2011) Reliability of transient elastography for the detection of fibrosis in non-alcoholic fatty liver disease and chronic viral hepatitis. J Hepatol 54: 64-71. [Crossref]

19. Leong K GR, Sood S, Nadenaum D, Gorelik A, Nicoll A (2017) Liver stiffness by ARFI does not correlate with decompensation and portal hypertension in patients with cirrhosis. J Dig Disord Diagn 1: 28-37.

20. Marcellin P, Asselah T, Boyer N (2002) Fibrosis and disease progression in hepatitis C. Hepatology 36: S47-56. [Crossref]

21. Ellis EL, Mann DA (2012) Clinical evidence for the regression of liver fibrosis. $J$ Hepatol 56: 1171-1180. [Crossref]

22. Xiao G, Yang J, Yan L (2015) Comparison of diagnostic accuracy of aspartate aminotransferase to platelet ratio index and fibrosis-4 index for detecting liver fibrosis in adult patients with chronic hepatitis B virus infection: a systemic review and metaanalysis. Hepatology 61: 292-302. [Crossref]

23. McPherson S, Stewart SF, Henderson E, Burt AD, Day CP (2010) Simple non-invasive fibrosis scoring systems can reliably exclude advanced fibrosis in patients with nonalcoholic fatty liver disease. Gut 59: 1265-1269. [Crossref]

24. Shaheen AA, Wan AF, Myers RP (2007) FibroTest and FibroScan for the prediction of hepatitis C-related fibrosis: a systematic review of diagnostic test accuracy. Am J Gastroenterol 102: 2589-2600. [Crossref]

25. Sheth SG, Flamm SL, Gordon FD, Chopra S (1998) AST/ALT ratio predicts cirrhosis in patients with chronic hepatitis C virus infection. Am J Gastroenterol 93: 44-48. [Crossref]

26. Boursier J, Vergniol J, Sawadogo A, Dakka T, Michalak S, et al. (2009) The combination of a blood test and Fibroscan improves the non-invasive diagnosis of liver fibrosis. Liver Int 29: 1507-1515. [Crossref]

Copyright: (C2018 Leong HK. This is an open-access article distributed under the terms of the Creative Commons Attribution License, which permits unrestricted use, distribution, and reproduction in any medium, provided the original author and source are credited. 